\title{
Energy Efficient Q-LEACH Protocols for Homogenous Wireless Sensor Networks
}

\author{
Ankit Kasana \\ Deptt of ECE, \\ Krishna Institute of \\ Engineering\& Technology \\ Ghaziabad, UP, India
}

\author{
Vibhav Kumar Sachan \\ Deptt of ECE, \\ Krishna Institute of \\ Engineering \& technology, \\ Ghaziabad, UP India
}

\author{
Nikhil Kumar Singh \\ Deptt of ECE, \\ Krishna Institute of \\ Engineering\& Technology \\ Ghaziabad, UP, India
}

\begin{abstract}
Wireless Sensor Network is web of sensors node, which has limiting energy .The main issue to design WSNs is, maximum Lifetime and optimal the energy used in network. Different approaches based upon clustering are proposed for optimum functionality. Quadrature-LEACH (Q-LEACH) for homogenous networks is examined with two gateways which is placed in the sensing area, which is used to enhances network life-time and optimal utilization of resources. Performance analysis and compared statistic results show that our proposed protocol perform well in terms of energy consumption and network lifetime.
\end{abstract}

\section{Keywords}

Energy efficient, Q- LEACH, Sensor Node

\section{INTRODUCTION}

Wireless Sensor Networks are mostly use for monitoring remote fields and critical conditions which are out of range from humans' perspective. For optimal distribution of energy among sensor nodes, in order to enhance network life time, suitable protocols and applications should be developed. Network lifetime can be defined as the time elapsed until the first node (or the last node) in the network depletes its energy (dies).Energy consumption in a sensor node can be due to either "useful" or "wasteful" sources. Use full energy consumption can be due to (i) transmitting/receiving data. (iii)processing query requests, and (iii) forwarding queries/data to neighboring nodes. Wasteful energy consumption can be due to (i) idle listening to the media. (ii) Retransmitting due to packet collisions, (iii) overhearing, and (iv) generating/handling control packets.

On the basis of energy distribution among sensor nodes, WSNs can be classified into homogenous and heterogeneous networks. Some clustering protocols such as LEACH [1], Power-Efficient Gathering in Sensor Information System (PEGASIS) [2], and Hybrid Energy-Efficient Distributed Clustering (HEED) [3] are defined for homogenous networks. Whereas, stable Election Protocol (SEP) [4] and Distributed Energy-Efficient Clustering (DEEC) [5] deal with heterogeneous networks.

Sensor networks are deployed for long term monitoring of fields and are desired to continue working without abrupt changes. Moreover, it is also desired to obtain global knowledge continuously i.e., better coverage of area should be

obtained. Considering above mentioned needs new approach Q-LEACH with M-GEAR [8]. are designed which improves network efficiency and enhance the network lifetime.

\subsection{System Model}

Sensor networks consisting of nodes with limited battery power and wireless communications are deployed to collect useful information from the field. Gathering sensed information in an energy efficient manner is critical to operate the sensor network for a long period of time. Fig. 1 describes optimal approach of load distribution among sensor nodes. Moreover, it also presents an idea of efficient clustering mechanism which yields significantly in better coverage of whole network. We deployed random nodes in a $100 \mathrm{~m} \times 100 \mathrm{~m}$ field. Based on location information, network is divided into four equal parts i.e, (a1, a2, a3, a4).Defining overall network area as below:

$$
A=a_{1}+a_{2}+a_{3}+a_{4}
$$

$a_{n}=A\left(x_{m}, y_{m}\right)$; where, $n=4$ and $m=100$. Hence, overall field is distributed as follow:

Two Gateway at $(25,50) ;(75,50)$ and Sink at outside the field at $(50,105)$.
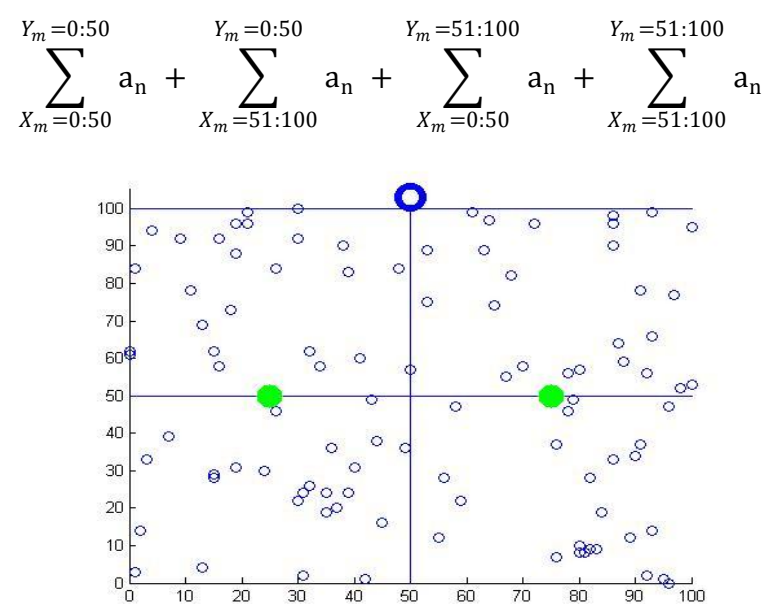

Figure (1): System Model [100m $\times 100 \mathrm{~m}$ area]

Portioning of network into quadrants yields in efficient energy utilization of sensor nodes. Through this division optimum positions of $\mathrm{CHs}$ are defined. Moreover, transmission load of other sending nodes is also reduced. For a Sensor Network we make the following assumptions:

- We deploy the Sink far away from the sensing field. Sensor nodes and the BS are stationary after deployment.

- A gateway node is deployed in the same network field. 
- Gateway node is stationary after deployment and rechargeable.

- Homogeneous sensor nodes with same computational and sensing capabilities are used.

- The energy cost for transmitting a packet depends on the distance of transmission.

- Each sensor node is assigned with a distinctive identifier (ID).

We use first order radio model [13],[14]. This model represents the energy dissipation of sensor nodes for transmitting, receiving and aggregating data. The transmitter dissipates more energy then receiver as it requires more energy for the transmitter electronics and amplifier. On the other hand, in receiver, only electronic circuits dissipate energy, as shown in fig (2).

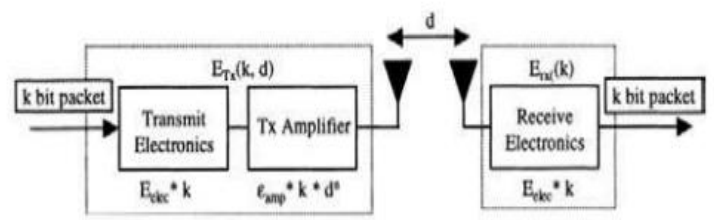

Figure (2): Radio Model

The energy required to transmit a data packet of $\mathrm{k}$ bits to a distance $\mathrm{d}$ and to receive a data packet of $\mathrm{k}$ bits, is given as:

$$
\begin{gathered}
E_{T x}(k, d)=E_{T x-\text { elec }}(k)+E_{T x-a m p}(k, d) \\
E_{T x}(k, d)=E_{\text {elec }} \times k+E_{\text {amp }} \times k \times d^{2} \\
E_{R x}(k)=E_{R x-\text { elec }}(k) E_{R x}(k)=E_{\text {elec }} \times k \\
E_{R x}(k)=E_{\text {elec }} \times k
\end{gathered}
$$

\section{RELATED WORK}

Azim et al. [12] introduced fixed LEACH algorithm depends on the relay of nodes. The battery power in WSN nodes is saved by using LEACH protocol and its different versions with clustering methods to reduce the energy used up by maintenance of more of the nodes in sleeping mode but give a good quality of service (QoS). But LEACH suffer on optimizing the life time of the network because of loosing huge amount of energy of sensor nodes elected as Cluster head during information transmissions. But relay node based technique used independent great relay nodes as heads to safeguard the energy in the small energy sensor nodes. These relay based technique also get trouble from a number of troubles like placement of relay nodes, blind spots and immature death of CHs. LEACH and modified LEACH except fixed LEACH, experience the problem of spending huge energy in the sensor nodes because of forming frequent cluster for each fixed time interval. Fixed LEACH solved the frequent clustering formation by build fixed clusters only once, but it wasted much energy and loss of information occurs because of premature death of cluster heads before expiring after a constant number of times. Therefore, LEACH-F can be used after the death of all the relay nodes, and the round time of fixed LEACH can be adjusted dynamically to decrease the probability of premature death of $\mathrm{CHs}$.

Wang and Yong [8] introduced cluster head selection by the pseudo cluster technique. Load monitor and Load leisure technique is used to equilibrium the load and stability of the topology of the network. In that Simulation $r$ LEACH-P Protocol effectively improve energy utilization efficiency, lengthens network lifetime and balances network load. Bhadeshiya et al.[9] proposed a routing scheme to select 4 or $5 \%$ of constant number of clusters in WSN depends on the residual energy.

$\mathrm{Mu}$ Tong et al. [10] introduced LEACH-B algorithm technique to balance the number of cluster heads depends on the residual energy of the nodes. Initial $\mathrm{CH}$ selection is depends on simple LEACH algorithm technique and from the second round LEACH-B is utilised. LEACH-B is a near optimal routing technique. LLEACH is a modified LEACH proposed by Qian Liao to choose $\mathrm{CHs}$ depends on residual energy and location identification of nodes and optimize the threshold for electing cluster-head. Cost function is designed to get better the optimal cluster-head selection. The experimental results proved L-LEACH balances the node energy better than LEACH.

Farooq et al. [11] proposed Multi-hop Routing with Low Energy Adaptive Clustering Hierarchy (MRLEACH) protocol for WSN.MR-LEACH divides the whole coverage area of the network into various layers of clusters. Cluster heads in one layer coordinate with the $\mathrm{CH}$ of neighbouring layers to passed the sensor's data to the central base station. Non Cluster head nodes link with cluster heads depends on the Received Signal Strength Indicator (RSSI).The communication of nodes is coordinated by a central Base Station (BS) which defines the Time Division Multiple Access (TDMA) scheme for every cluster-head. Usually, BS uses the Top layers of CHs to perform as super cluster heads for Cluster Heads at the lower layer. Thus, MRLEACH utilise multi-hop routing process from clusterheads to a central station to accumulate energy, in contrast with the LEACH protocol. When estimate the performance, it is proved that MRLEACH yields important improvement when comparing to the LEACH protocol and energy efficient routing for WSN.

A centralized version of LEACH, LEACH-C, is introduced. Unlike LEACH, sensor nodes are self-configure itself into clusters, LEACH-C use the base station for cluster configuration. During the setup phase of LEACH-C, the base station receives data regarding the location and energy level of every node in the sensor network. Using this information, the base station discovers a predetermined number of cluster heads and configures the sensor network into clusters. The cluster groupings are chosen to decrease the energy required for non-cluster- head nodes to broadcast their information to their respective cluster heads.

B. Manzoor [15] proposed Quadrature-Low Energy Adaptive Cluster Hierarchy (Q-LEACH)for homogenous networks. Divides the whole network in to four part (or in to the quadrature).Through this division optimum positions of $\mathrm{CHs}$ are defined. Moreover, transmission load of other sending nodes is also reduced. Each $\mathrm{CH}$ selection base upon on probability [1].Non-CHs nodes will locate themselves in specified area they belong to. Then they will search for all possible $\mathrm{CHs}$ and on the basis of RSSI they will start association. This process will continue until association phase comes to an end.

\subsection{Lifetime of a Sensor Network}

The definition of the lifetime of a sensor network is determined by the kind of service it provides. Hence, three new approaches of defining lifetime are proposed. In some cases it is necessary that all nodes stay alive as long as 
possible, since network quality decreases considerably as soon as one node dies. Scenarios for this case include intrusion or fire detection. In these scenarios it is important to know when the first node dies. The new metric First Node Dies (FND) denotes an estimated value for this event for a specific network configuration. Furthermore, sensors can be placed in proximity to each other. Thus, adjacent sensors could record related or identical data. Hence, the loss of a single or few nodes does not automatically diminish the quality of service of the network. In this case the new metric Half of the Nodes Alive (HNA) denotes an estimated value for the half-life period of a sensor network. Finally, the metric Last Node Dies (LND) gives an estimated value for the overall lifetime of a sensor network for a cluster-based algorithm like LEACH the metric LND is not interesting since more than one node is necessary to perform the clustering algorithm. Hence, we limit the discussion of algorithms in this paper to the metrics FND and HNA.

\subsection{Problem Formulation}

In conventional LEACH cluster are arbitrary in size and some of the cluster members are located far away. Due to this dynamic cluster formation farther nodes suffers through high energy drainage and thus, network performance degrades. Whereas, in Q-LEACH network is partitioned into sub-sectors and hence, clusters formed within these sub-sectors are more deterministic in nature. Therefore, nodes are well distributed within a specific cluster and results in efficient energy drainage .Concept of randomized clustering as given in [Algorithm1] for optimized energy drainage is applied in each sector. Assigning $\mathrm{CH}$ probability $\mathrm{P}=0.05$ we start clustering process. In every individual round nodes decides to become $\mathrm{CH}$ based upon $\mathrm{P}$ and threshold $\mathrm{T}(\mathrm{n})$ given in [Algorithm1] as:

\section{Algorithm 1}

1. Begin

2. if node $\varepsilon \mathrm{G} \rightarrow \mathrm{G}$ = node which did not become $\mathrm{CHs}$ in current $\mathrm{EPOCH}$ then

3. if (Node_belongs_to $==$ 'areaA') then

4. if $\left(N M B E R O F C H s<=\left(\frac{N}{K}\right)\right)$ then

5. TEMP=random number $(0-1)$

6. If $\left(\right.$ temp $\left.<=\frac{P}{1-P\left(r, \bmod ^{1} / P\right)}\right)$ then

7. node=CH_A

8. Number_of_CHs $=$ Number_of_CHs +1

9. end if

10. else if (node_belongs_to $==$ 'area B') then

11. REPEAT STEP $4: 8$

12. else if (node_belongs_to $==$ 'area $\mathrm{C}$ ') then

13. REPEAT STEP $4: 8$

14. else if (node_belongs_to $==$ 'area $D$ ') then

15. REPEAT STEP $4: 8$

16. end if

17. end if

18. end if

Algorithm.1 defines $\mathrm{CHs}$ selection mechanism. Overall network is divided into four areas as: Area A,B,C and D. Initially each node decides whether or not to become a $\mathrm{CH}$. Node chooses a random number between 0 and 1 . If this number is less then certain threshold $\mathrm{T}(\mathrm{n})$, and condition for desired number of $\mathrm{CHs}$ in a specific area is not met, then the node becomes a $\mathrm{CH}$. Similarly the same process continues for rest of the sectors and optimum number of clusters is formed. After decision of clusters, nodes must tell $\mathrm{CHs}$ about their association. On the basis of gathered information from attached nodes, guaranteed time slots are allocated to nodes using Time Division Multiple Access (TDMA) approach. Moreover this information is again broadcasted to sensor nodes in the cluster.

After selection of all CHS, Each CH communicate with which has shortest distance either sink or gateway. Gateway node not a sink, although it directly communicate with sink. Figure 3 show the position of the sink and gateway. Gateway is the high energy node in the network for those which are away from the sink node also along with this the nodes with high energy. Gateway has rechargeable or easy to replace battery. We use the same radio model [3] with $E_{\text {elec }}=50 \mathrm{~nJ} /$ bit as the energy being dissipated to run the transmitter or receiver circuitry and $\varepsilon_{\mathrm{amp}}=100 \mathrm{pJ} / \mathrm{bit} / \mathrm{m}^{2}$ as the energy dissipation of the transmission amplifier. Transmission $\left(\mathrm{E}_{\mathrm{Tx}}\right)$ and receiving costs $\left(\mathrm{E}_{\mathrm{Rx}}\right)$ are calculated as follows:

$$
\begin{aligned}
& E_{T X}(k, d)=E_{\text {elec }} k+\varepsilon_{\text {amp }} k d^{\lambda} \\
& E_{R x}=E_{\text {elec }} k
\end{aligned}
$$

With $\mathrm{k}$ as the length of the message in bits, $\mathrm{d}$ as the distance between transmitter and receiver node and $\lambda$ as the path-loss exponent $(2 \geq \lambda)$.

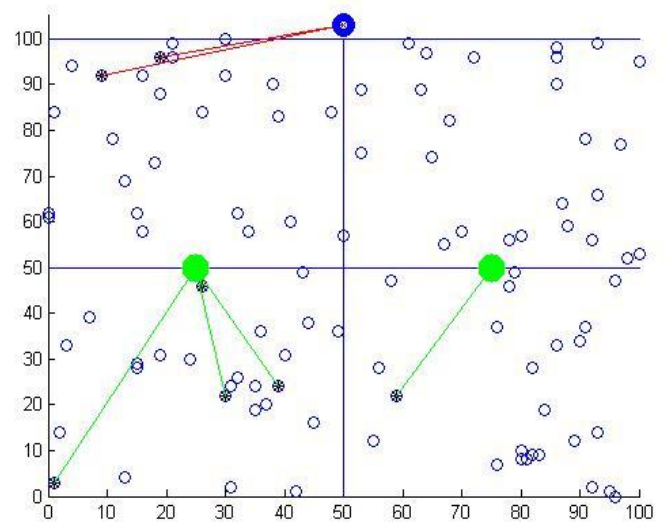

Figure (3): Working model

\section{SIMULATION RESULTS}

In this section, we discuss simulations results of Q-LEACH with Gateway for WSNs. Moreover, MATLAB is used as a simulation tool.We deploy a random network of 100 nodes with initial energy $=0.5 \mathrm{j}$ in filed with dimensions of $100 \mathrm{~m}$ $\times 100 \mathrm{~m}$. In simulated scenario Sink is placed far away from the network field. We evaluated performance of our proposed strategy on the basis of certain parameter network number of alive node, number of died node, number of packet send to Gateway and Sink.

\section{CONCLUSION}

In this paper, We introduce the high energy node(Gateway node) in the network for those which are away from the sink node also along with this the nodes with high energy will be include ,the main process of communication to enhancing the lifetime of the nodes. The simulation result shows the life time of sensor is twice than the traditional Q-LEACH [1] approach. In traditional Q-LEACH approach first node is dead between the 2000-2200 rounds but in our approach is around 4000-4200 round. 

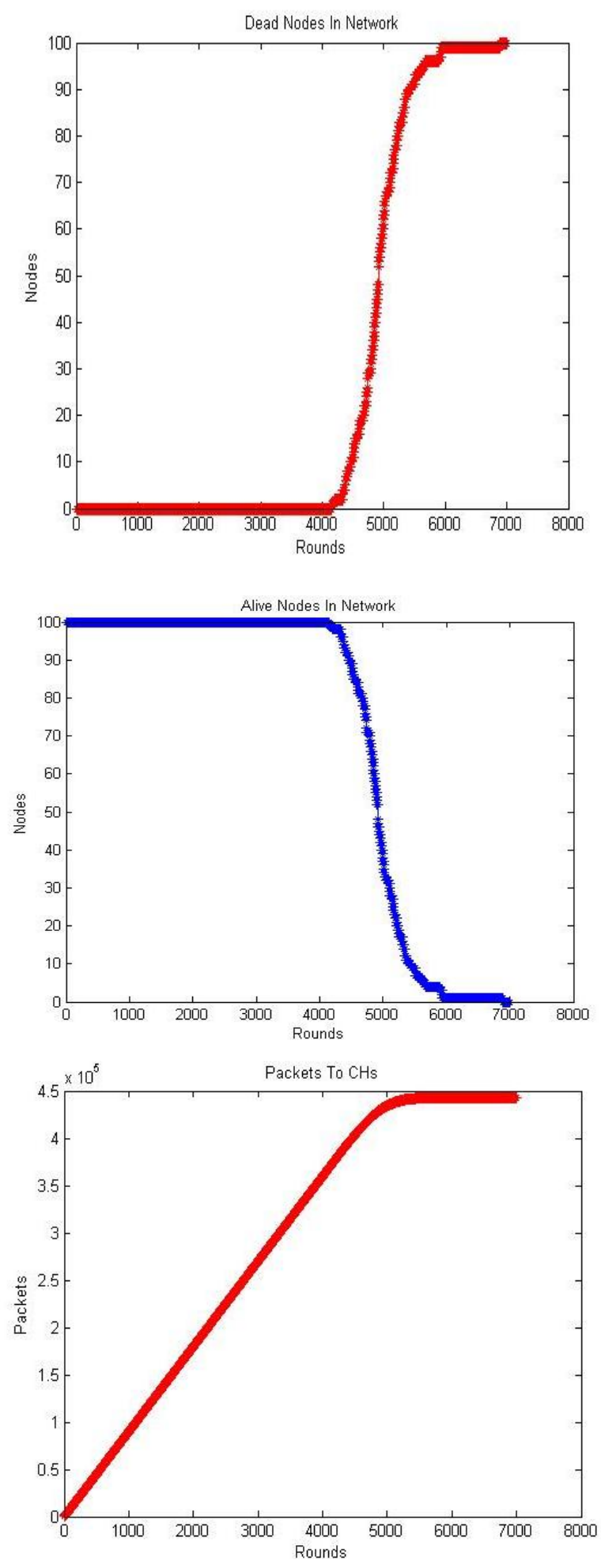

\section{REFERENCES}

[1] M. Ishfaq; Q-LEACH: A New Routing Protocol for WSNs. The 4th International Conference on Ambient Systems, Networks and Technologies (ANT 2013), Procedia Computer Science; Volume 19, 2013, Pages 926-931

[2] S. Lindsey and C. Raghavendra, Pegasis: Power-efficient gathering in sensor information systems, in Aerospace conference proceedings, 2002. IEEE, vol. 3, pp. 31125 , IEEE, 2002.
[3] O. Younis and S. Fahmy, Heed: a hybrid, energyefficient, distributed clustering approach for ad hoc sensor networks, Mobile Computing, IEEE Transactions on, vol. 3, no. 4, pp. 366379, 2004.

[4] G. Smaragdakis, I. Matta, and A. Bestavros, Sep: A stable election protocol for clustered heterogeneous wireless sensor networks, tech. rep., Boston University Computer Science Department, 2004.

[5] L. Qing, Q. Zhu, and M. Wang, Design of a distributed energy-efficient clustering algorithm for heterogeneous wireless sensor networks, Computer communications, vol. 29, no. 12, pp. 22302237, 2006.

[6] Y. Yu, R. Govindan, and D. Estrin, Geographical and energy aware routing: A recursive data dissemination protocol for wireless sensor networks, tech. rep., Citeseer, 2001

[7] A. Elrahim, H. Elsayed, S. Ramly, and M. Magdy, An energy aware wsn geographic routing protocol, Universal Journal of Computer Science and Engineering Technology, vol. 1, no. 2, pp. 105111, 2010.

[8] Wangang Wang and Yong Peng. 2013. "LEACH Algorithm Based on Load Balancing", TELKOMNIKA Indonesian Journal of Electrical Engineering, Vol. 11, No. 9, pp. 5329-5335.

[9] J. R. Bhadeshiya and S. Vora. 2012. "A Reformed Cluster-Head of LEACH Protocol and Performance Analysis with Conventional Routing Protocol for WSN", Journal of Information, Knowledge and Research in Electronics and Communication Engineering, Vol. 2, No. 2, pp. 812-816.

[10] Mu Tong and Minghao Tang. 2010. "LEACH-B: An Improved LEACH Protocol for Wireless Sensor Network", Proceedings of 6th International Conference on Wireless Communications Networking and Mobile Computing, pp. 1-4.

[11] Farooq M. O., Dogar A. B. and Shah G. A. 2010. "MRLEACH: Multi-hop Routing with Low Energy Adaptive Clustering Hierarchy", Proceedings of 4th International Conference on Sensor Technologies and Applications, pp. 262-268.

[12] Akramul Azim and Mohammad Mahfuzul Islam. 2012. "A Relay Node Based Hybrid Low Energy Adaptive Clustering Hierarchy for Wireless Sensor Networks", International Journal of Energy, Information and Communications, Vol. 3, No. 3, pp. 41-54.

[13] Heinzelman, Wendi Rabiner, AnanthaChandrakasan, and HariBalakrishnan."Energy-efficient communication

[14] Nikhil Kumar Singh, Ankit Kasana and Vibhav Kumar Sachan. Enhancement in lifetime of sensor node using Data Reduction Technique in Wireless Sensor Network. International Journal of Computer Applications 145(11):1-5, July 2016

[15] Himanshu Sharma, Vibhav Kumar Sachan and Syed Akhtar Imam. Article: Energy Efficiency of the IEEE 802.15.4 Standard in Wireless Sensor Networks: Modeling and Improvement Perspectives. International Journal of Computer Applications 58(9):12-19, November 2012 


\section{AUTHOR PROFILE}

Mr. Ankit kasana has received his B.Tech in Electronic and Communication engineering from Collage \& Rural Technology (2011).affiliated to Gautam buddh Technical University (GBTU) lucknow, India and pursuing M.Tech in (ECE) from KIET Ghaziabad affiliated to AKTU U.P Lucknow. His main Research area enhancement in life time of sensor node by Energy efficient protocol.
Mr. Nikhil Kumar Singh has received his B.Tech degree in Electronic and Communication engineering from Prasad Institute of Technology (2010) affiliated to Gautam Buddh Technical Uneversity (GBTU) Lucknow, India and pursuing M.Tech in Electronics and Communication engineering from Krishna Institute of Engineering Technology Ghaziabad, affiliated to AKTU (Abdul Kalam Technical University) Uttar Pradesh Lucknow. His main Research areas of interest are enhancement in life time of sensor node using Huffman Coding e.t.c. 